\title{
PICK1-ICA69 Heteromeric BAR Domain Complex Regulates Synaptic Targeting and Surface Expression of AMPA Receptors
}

\author{
Mian Cao, ${ }^{1}$ Junyu Xu, ${ }^{1}$ Chong Shen, ${ }^{1}$ Chuen Kam, ${ }^{1}$ Richard L. Huganir, ${ }^{2}$ and Jun Xia ${ }^{1}$ \\ ${ }^{1}$ Department of Biochemistry, The Hong Kong University of Science and Technology, Clear Water Bay, Kowloon, Hong Kong, China, and ${ }^{2}$ Department of \\ Neuroscience and Howard Hughes Medical Institute, Johns Hopkins University School of Medicine, Baltimore, Maryland 21205
}

\begin{abstract}
The trafficking of AMPA-type glutamate receptors to and from synapses is an important mechanism underlying synaptic plasticity, a cellular model of learning and memory. PICK1 (protein interacts with C-kinase 1) is a peripheral membrane protein that interacts with AMPA receptors and regulates their trafficking. PICK1 contains a PDZ (PSD-95/Dlg/Z01) domain and a BAR (Bin/amphiphysin/Rvs) domain. The PDZ domain of PICK1 interacts with the intracellular C-terminal tails of AMPA receptors, while the BAR domain binds to lipid membranes. Both the AMPA receptor interaction and the lipid binding of PICK1 are important to AMPA receptor trafficking and synaptic plasticity. Here, we identified ICA69 (islet cell autoantigen $69 \mathrm{kDa}$ ), another BAR-domain-containing protein, as the major binding partner of PICK1. Over three-fourths of ICA69 and PICK1 associate with each other in the brain. The BAR domain of ICA69 also binds to liposomes and forms heteromeric BAR domain complexes with PICK1. ICA69 coexpresses with PICK1 in different tissues and at various developmental stages. In neurons, although ICA69 colocalizes well with PICK1 in cell bodies and dendrites, it is surprisingly absent from synapses, where PICK1 is enriched. Furthermore, overexpression of ICA69 redistributes PICK1 from synapses to dendrites. ICA69 also disrupts the PICK1-induced clustering of AMPA receptors and reduces synaptic targeting and surface expression of the receptors. ICA69 regulates AMPA receptor trafficking by forming heteromeric BAR domain complexes with PICK1 and preventing formation of PICK1 homomeric complexes. Together, our results suggest that the switch from ICA69-PICK1 heteromeric complexes to PICK1-PICK1 homomeric complexes could be an important mechanism regulating synaptic targeting and surface expression of AMPA receptors.
\end{abstract}

Key words: PICK1; ICA69; BAR domain; AMPA receptor; synaptic plasticity; protein trafficking

\section{Introduction}

The AMPA-type glutamate receptors mediate the majority of fast excitatory synaptic transmissions in the CNS. Trafficking of AMPA receptors to and from synapses is a key molecular event underlying synaptic plasticity such as long-term potentiation and long-term depression (LTD) (Malinow and Malenka, 2002; Collingridge et al., 2004). The trafficking of AMPA receptors is regulated by a number of associated proteins (Barry and Ziff, 2002; Song and Huganir, 2002; Bredt and Nicoll, 2003; Henley, 2003). PICK1 (protein interacts with C-kinase 1) is a protein that directly interacts with AMPA receptors and regulates their trafficking. PICK1 contains a PDZ (PSD-95/Dlg/ZO1) domain and a BAR (Bin/amphiphysin/Rvs) domain, and its PDZ domain interacts with the $\mathrm{C}$ termini of AMPA receptors (Xu and Xia, 2007).

Multiple lines of evidence indicate that PICK1 is a key regula-

Received May 4, 2007; revised Sept. 21, 2007; accepted Sept. 25, 2007.

This work was supported in part by grants from the Research Grants Council of the Hong Kong Special Administrative Region, China (HKUST6144/03M, HKUST6130/04M, and N_HKUST605/07). We thank W. Tung, C. He, and K. Lai for their excellent technical assistance and Dr. D. Banfield for critical reading of this manuscript.

Correspondence should be addressed to Dr. Jun Xia, Department of Biochemistry, the Hong Kong University of Science and Technology, Clear Water Bay, Kowloon, Hong Kong, China. E-mail: jxia@ust.hk.

D0I:10.1523/JNEUROSCI.2040-07.2007

Copyright $\odot 2007$ Society for Neuroscience $\quad 0270-6474 / 07 / 2712945-12 \$ 15.00 / 0$ tor of AMPA receptor trafficking and synaptic plasticity. PICK1 has been found to induce clustering of GluR2, a subunit of AMPA receptors, and increase the number of AMPA receptors at synapses (Dev et al., 1999; Xia et al., 1999; Terashima et al., 2004; Jin et al., 2006). PICK1 also regulates surface expression of AMPA receptors in a protein kinase-dependent manner (Matsuda et al., 1999; Chung et al., 2000; Perez et al., 2001; Terashima et al., 2004). Disrupting the interaction between PICK1 and AMPA receptors inhibits LTD in both the cerebellum and hippocampus (Matsuda et al., 2000; Xia et al., 2000; Kim et al., 2001). In PICK1 knock-out mice, LTD in cerebellar Purkinje neurons is completely abolished (Steinberg et al., 2006). In addition, PICK1 knock-out mice have deficiencies in calcium-permeable AMPA receptor-mediated plasticity, a process that also requires the interaction of PICK1 with GluR2 (Gardner et al., 2005; Liu and Cull-Candy, 2005).

BAR domains are banana-shaped dimers that bind to lipid membranes and initiate vesicle formation by sensing membrane curvature or actively bending membranes (Peter et al., 2004; Dawson et al., 2006; Ren et al., 2006). The BAR domain of PICK1 was recently demonstrated to bind to lipids (Jin et al., 2006). Lipid binding is required for PICK1's synaptic targeting and PICK1-mediated AMPA receptor trafficking. Lipid-binding- 
deficient PICK1 loses its ability to cluster GluR2 and no longer targets AMPA receptors to synapses. Moreover, lipid-bindingdeficient PICK1 is unable to regulate the surface expression of AMPA receptors and impairs expression of LTD (Jin et al., 2006). Whereas wild-type PICK1 rescued the cerebellar LTD deficiency in PICK1 knock-out mice, lipid-binding-deficient PICK1 was unable to do so (Steinberg et al., 2006). These results suggest that with its BAR domain binding to membranes, PICK1 may tether AMPA receptors as cargos to membrane microdomains that are designated to form trafficking vesicles.

To gain more insight into the molecular machinery that is responsible for PICK1-mediated receptor trafficking, we performed coimmunoprecipitation coupled with mass spectrometry analysis to identify PICK1-associated proteins. Here, we report that ICA69 (Islet Cell Autoantigen $69 \mathrm{kDa}$ ), another BAR domain-containing protein, forms tight heteromeric BAR domain complexes with PICK1 and regulates AMPA receptor trafficking.

\section{Materials and Methods}

cDNA cloning and fusion protein purification

Rat ICA69 cDNA was obtained from cDNA library using a combination of library screening and PCR amplification and was verified by sequencing. ICA-BAR (amino acids 1-276) and ICAC (amino acids 257-480) fragments were generated by PCR from full-length rat ICA69 cDNA. ICA69 full-length, ICA-BAR, and ICAC cDNAs were then subcloned in frames into myc-pRK5, pEGFP-C3, pPC86, and pET-MBP vectors by SalI/NotI. All PICK1 and GluR2 constructs used here were described previously (Jin et al., 2006). To produce fusion proteins, cDNA constructs were transformed into Escherichia coli BL21 cells and induced with isopropyl- $\beta$-D-thiogalactopyranoside. Maltose-binding protein (MBP) fusion protein was affinity purified by amylose resin (New England Biolabs, Beverly, MA), according to the instructions of the manufacturer. Fusion protein concentrations were determined by Coomassie assays (Pierce, Rockford, IL).

\section{Antibodies}

Anti-ICA69 rabbit polyclonal antibodies used in immunostaining and coimmunoprecipitation were generated against two antigens: a peptide corresponding to residues 468-480 (IGKTDKEHELLNA) of rat ICA69 and a His-tagged fusion protein of rat ICA69 (amino acids 215-480). Antibody specificity was tested by preblocking with antigens. AntiPICK1 guinea pig polyclonal antibody used in immunostaining was generated against the C-terminal 100 aa of mouse PICK1 (PC100). AntiPICK1 rabbit polyclonal antibodies used in coimmunoprecipitation were generated against the N-terminal 29 aa (PN29) or C-terminal 100 aa (PC100) of mouse PICK1. Anti-GluR2/3 rabbit polyclonal antibody was raised using C-terminal 20 aa of GluR2 as antigen. Anti-green fluorescent protein (GFP) rabbit polyclonal antibody was generated using GFP fusion protein as the antigen. Anti-hemagglutinin (HA) mouse monoclonal antibody was purchased from Sigma (St. Louis, MO). Anti-myc mouse monoclonal antibody was purchased from Developmental Studies Hybridoma Bank (Iowa City, IA) (9E10). Horseradish peroxidase (HRP)-labeled secondary antibody was purchased from GE Healthcare (Piscataway, NJ). HRP-labeled protein A was purchased from Bio-Rad (Hercules, CA). Secondary antibodies labeled with Alexa Fluor-488 or 647 were purchased from Invitrogen (Carlsbad, CA). Secondary antibodies labeled with rhodamine red $\mathrm{X}$ were from Jackson ImmunoResearch (West Grove, PA).

\section{Purification and identification of PICK1-binding proteins}

Mouse brains were homogenized in $10 \mathrm{~mm}$ Tris-HCl buffer with $320 \mathrm{~mm}$ glucose, $\mathrm{pH} 7.4$, and solubilized in TBS buffer with $2 \%$ Triton X-100 for $2 \mathrm{~h}$. The brain samples were then subjected to high-speed centrifugation at $12,000 \mathrm{rpm}$ for $20 \mathrm{~min}$, and the supernatants were incubated with purified rabbit PICK1 N29 antibody for at least $4 \mathrm{~h}$ at $4^{\circ} \mathrm{C}$. For peptideblock control, antibodies were preincubated with PICK1 N29 peptide for $2 \mathrm{~h}$ before combining with brain samples. Protein A beads were added into the samples and incubated for $\sim 2 \mathrm{~h}$ to pull down the protein complex. The bound proteins were resolved by SDS-PAGE and stained with GelCode blue (\#24590; Pierce). The band around $70 \mathrm{kDa}$ from nonblocked sample was cut out and subjected for mass spectrometry analysis.

\section{Yeast two-hybrid assay}

For the interacting domain mapping, PICK1 full length (amino acids 1-416), PICK1-PDZ (amino acids 1-146), PICK1-BAR (amino acids 147-358), and PICK1-C (amino acids 358-416) were subcloned in frame into pDBLeu vector, which contains the GAL4 DNA-binding domain. ICA69 full length (amino acids 1-480), ICA-BAR (amino acids 1-276), and ICAC (amino acids 257-480) were subcloned into pPC86 vector, which contains the GAL4 activation domain (Xia et al., 1999). Two constructs from different groups were cotransformed into HF7c yeast cells in pairs and grown on double-minus plates lacking leucine and tryptophan. The positive colonies were then patched onto triple-minus plates that lacking leucine, tryptophan, and histidine. Positive clones were scored by both growth and blue assays.

\section{Coimmunoprecipitation}

In vitro coimmunoprecipitation. To test PICK1-ICA69 interaction, HEK 293 T cells were cotransfected with GFP-PICK1 and myc-ICA69 (or GFPICA69 and myc-PICK1) $3 \mathrm{~d}$ before coimmunoprecipitation. Anti-GFP sera $(1 \mu \mathrm{l})$ were preincubated with $40 \mu \mathrm{l}$ of protein A beads (GE Healthcare) for $1 \mathrm{~h}$ at $4^{\circ} \mathrm{C}$. At the same time, transfected $293 \mathrm{~T}$ cell lysates $(1 \mathrm{ml}$ of each) were extracted by $1 \%$ Triton X-100 in PBS following top-speed centrifugation for $20 \mathrm{~min}$ at $4^{\circ} \mathrm{C}$. Antibody/protein A beads complexes were then incubated with cell lysates for at least $2 \mathrm{~h}$ at $4^{\circ} \mathrm{C}$. The beads were washed once with cold PBS plus $1 \%$ Triton X-100, twice with cold PBS plus $1 \%$ Triton $\mathrm{X}-100$ plus $500 \mathrm{~mm} \mathrm{NaCl}$, and three times with cold PBS. After washing, beads were eluted with $1 \times$ SDS sample buffer and analyzed by SDS-PAGE and immunoblotted with anti-myc or anti-GFP antibody. To test ICA69-GluR2 interaction, the effect of ICA69 on PICK1-GluR2 interaction, or PICK1 self-association, DNA constructs in different combinations (myc-ICA69 and GluR2; myc-ICA69, GFPPICK1, and GluR2; myc-ICA69, myc-PICK1, and GFP-PICK1) were cotransfected into $293 \mathrm{~T}$ cells, respectively, and coimmunoprecipitation was performed to pull down overexpressed PICK1 or ICA69 proteins as mentioned earlier.

In vivo coimmunoprecipitation. Approximately $500 \mu \mathrm{g}$ of rat brain homogenates were solubilized by $1 \%$ Triton X-100 plus protease inhibitor mixture for $30 \mathrm{~min}$ at $4^{\circ} \mathrm{C}$ and spun down at maximum speed for 20 $\min$ at $4^{\circ} \mathrm{C}$. The supernatant was used for immunoprecipitation (IP). Approximately $10 \mu \mathrm{g}$ of affinity-purified PICK1 antibody was preincubated with $40 \mu \mathrm{l}$ of $1: 1$ slurry of protein A Sepharose for $1-2 \mathrm{~h}$ at $4^{\circ} \mathrm{C}$. The antibody-protein A beads complexes were spun down at $2000 \mathrm{rpm}$ for 2 $\min$ at $4^{\circ} \mathrm{C}$. The clarified supernatant of brain homogenates was then added into the beads, and the mixtures were incubated for $2-3 \mathrm{~h}$ at $4^{\circ} \mathrm{C}$. The mixtures were washed once with $1 \%$ Triton X-100 in cold TBS, twice with $1 \%$ Triton X-100 in cold TBS plus $500 \mathrm{~mm} \mathrm{NaCl}$, and three times with cold TBS. The immunoprecipitates were resolved by SDS-PAGE and analyzed by Western blotting. HRP-coupled protein A, which eliminates the IgG background in IP samples, was used to detect PICK1. For consecutive co-IP, both anti-PICK1 and anti-ICA69 sera were coupled with protein A beads. The supernatant after each IP was collected and reincubated with newly prepared antibody-coupled protein A beads for $2-3 \mathrm{~h}$. This process was repeated two to three times to completely pull down all of PICK1 or ICA69 proteins from brain homogenates. IP and co-IP efficiencies were determined by densitometry analysis, and the ratio was calculated as AIP (supernatant after IP)/INPUT (supernatant before IP).

\section{Tissue blotting and developmental profile}

For tissue blotting, different tissues were dissected out from rat separately and homogenized using homogenate buffer $(10 \mathrm{~mm}$ Tris- $\mathrm{Cl}, 320 \mathrm{~mm}$ sucrose, $\mathrm{pH}$ 7.4) to obtain the total proteins. For developmental profile, brains were dissected out from B6 mice at different developmental stages [from embryonic day 15 (E15) to postnatal day 90 (P90)]. Protein concentration was determined by Coomassie assay (Pierce). Equal amounts 
A

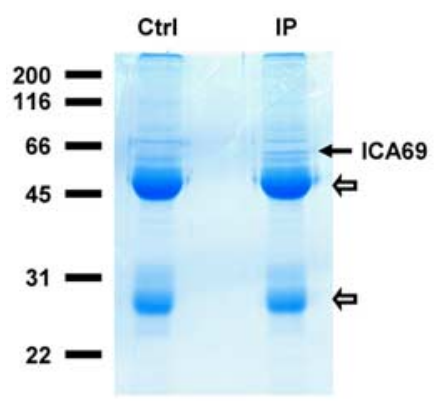

C
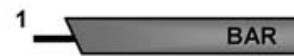

1 MSGHKCYSWE LQDRFAQDKS VVNKMQQKYW ETKQAFIKAT 41 GKKEDEHVVA SDADLDAKLE LFHSIORTCL DLSKATVLYO 81 KRICFLSQEE NELGKFLRSQ GFQDKTRAGK MMQATGKALC 121 ESSQQRLALR NPLCREHQEV ETFRHRAISD TWLTVNRMEQ 161 YRTEYRGALL WMKDVSQELD PDLYKQMEKF RKVQTQVRLA $\propto$ 201 KKNFDKLKMD VCQKVDLLGA SRCNLLSHML ATYQTTLLHF 241 WEKTSHTMAA IHESFKGYQP YEETTLKSLQ DPMKKLVEKE 281 GKKTSWRENR EAVAPEPROL ISLEDEHKDS STYKTEEGTS 321 VLSSVDKGSV HDTCSGPIDE LLDGKPEEAC LGPTAGTPEP 361 ESGDKDDLLL LNEIFSTSCL DEGEFSREWA AVFGDDRLKE 401 PAPMGAQGEP DPKPQIGSGF LPSQLLDQNM KDLQASLOEP S 441 AKAASDLTAW FSLFADLDPL SNPDAVGKTD KEHELLNA
ICA69

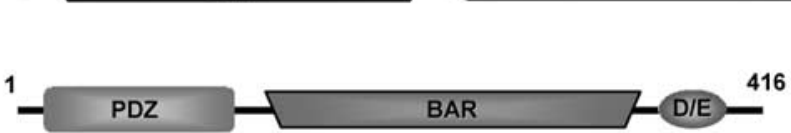

\begin{tabular}{|l|c|c|c|c|}
\hline & ICA69(1-480) & ICA-BAR(1-276) & ICAC(257-480) & pPC86 vector \\
\hline PICK1(1-416) & + & + & + & - \\
\hline PICK1-PDZ(1-146) & - & - & - & - \\
\hline PICK1-BAR(147-358) & + & + & - & - \\
\hline PICK1-C(358-416) & - & - & - & - \\
\hline pDBLeu vector & - & - & - & - \\
\hline
\end{tabular}

Figure 1. Identification of ICA69 as a major PICK1 binding partner and mapping of the interaction domain. $\boldsymbol{A}$, Mouse brain homogenate was immunoprecipitated by an anti-PICK1 antibody. In the control (Ctrl) IP, the anti-PICK1 antibody was blocked by antigenic peptides. The two hollow arrows indicate the lgG heavy chain and light chain. The solid arrow indicates the strongest band found in IP with the PICK1 antibody but not in the control IP. The band was cut out and identified as ICA69 by mass spectrometry. $\boldsymbol{B}$, The amino acid sequence of mouse ICA69. The shaded areas indicate the BAR domain and the $\mathbf{C}$-terminal domain (ICAC) of ICA69. The red letters indicate the eight peptides identified in the mass spectrometry analysis. $C$, Different regions of PICK1 in the pDBLeu vector and ICA69 in the PPC86 vector were transformed to yeast and grown on selective plates. Interactions were judged by both growth on selective plates and $\beta$-galactosidase blue assays. Positive and negative interactions are designated with "+" and"-," respectively.

of proteins $(\sim 20 \mu \mathrm{g} /$ lane $)$ were loaded to SDS-PAGE and analyzed by Western blotting using anti-PICK1 or anti-ICA69 antibodies.

\section{Liposome sedimentation assay}

The liposome sedimentation assay was performed by following the procedure described previously (Jin et al., 2006). Briefly, brain lipid extracts (Folch fraction I, Sigma B1502) were resuspended at $2 \mathrm{mg} / \mathrm{ml}$ in a buffer containing $20 \mathrm{~mm}$ HEPES, pH 7.4, $150 \mathrm{~mm} \mathrm{NaCl}$, and $1 \mathrm{~mm}$ DTT. Fusion proteins $(5 \mu \mathrm{M})$ were incubated with $0.6 \mathrm{mg} / \mathrm{ml}$ liposomes in $100 \mu \mathrm{l}$ of buffer for $15 \mathrm{~min}$ at $37^{\circ} \mathrm{C}$ and then spun at $140,000 \times g$ for $15 \mathrm{~min}$ at $4^{\circ} \mathrm{C}$ in a Beckman (Fullerton, CA) TLA100.1 rotor. Supernatants were saved, and the pellets were washed once with the same buffer and brought up to the same volume as the supernatant. The supernatant and the pellet proteins were subjected to SDS-PAGE and visualized by Coomassie stain.

\section{Cell culture and transfection}

HEK 293T cells were cultured in humidified atmosphere containing 5\% $\mathrm{CO}_{2}$ in MEM (Invitrogen) with $10 \%$ fetal bovine serum, $1 \mathrm{~mm}$ sodium pyruvate, $100 \mathrm{U} / \mathrm{ml}$ penicillin, and $100 \mu \mathrm{g} / \mathrm{ml}$ streptomycin and passed every $3 \mathrm{~d}$ when the cell confluence reached to $80-90 \%$. Calcium phosphate coprecipitation method was used on 293T cells for transient transfection, and medium was changed $9 \mathrm{~h}$ later completely. For neuronal culture, hippocampal neurons were prepared from embryonic day 18 rats (Sprague Dawley) and grown on coverslips coated with poly-L-lysine (Sigma). Hippocampal neurons were transfected by calcium phosphate coprecipitation at 5-7 $\mathrm{d}$ in vitro.

\section{Immunocytochemistry}

HEK 293 T cell staining. The cells were fixed $36-48 \mathrm{~h}$ after transfection by $4 \%$ paraformaldehyde plus $4 \%$ sucrose in PBS for 20 min at room tem-

perature. The cells were then permeabilized by $0.2 \%$ Triton $\mathrm{X}-100$ in PBS for 10 min at room temperature. After blocking with 10\% normal donkey serum (NDS) in PBS for $1 \mathrm{~h}$, the cells were incubated with primary antibody in 3\% NDS for $1 \mathrm{~h}$ at room temperature, followed by $1 \mathrm{~h}$ of incubation with fluorescence-conjugated secondary antibody (Jackson ImmunoResearch). After washing with PBS, the coverslips were mounted with Permafluor (Immunon). The cells were observed with a Nikon (Tokyo, Japan) Eclipse TE2000 inverted fluorescence microscope under a $60 \times$ plan Apochromatic oil lens (numerical aperture, 1.4). Pictures were taken by a monochrome cooled CCD camera (SPOT-RT; Diagnostic Instruments, Sterling Heights, MI) controlled by MetaMorph imaging acquisition software (Universal Imaging, Downingtown, PA). Images were processed by Adobe (San Jose, CA) Photoshop to adjust intensity and contrast, to select region of interest, and to overlay two images. All images were taken in monochrome gray scale and artificially colored for presentation.

Neuron staining. Day 17-21 neurons were fixed by $4 \%$ paraformaldehyde plus $4 \%$ sucrose in PBS for 15 min at $4^{\circ} \mathrm{C}$ and then permeabilized by $0.2 \%$ Triton $\mathrm{X}-100$ for $10 \mathrm{~min}$ at $4^{\circ} \mathrm{C}$. After blocking with $10 \%$ normal donkey serum for $>2 \mathrm{~h}$ at room temperature, the neurons were incubated with primary antibody in $3 \%$ NDS at $4^{\circ} \mathrm{C}$ overnight or $1 \mathrm{~h}$ at room temperature. For surface HA-GluR2 staining, neurons were first incubated with mouse anti-HA antibody for $1 \mathrm{~h}$ at room temperature before permeabilization. Fluorescent secondary antibodies were added for $1 \mathrm{~h}$ at room temperature. For F-actin staining, the neurons were further incubated with rhodamine-phalloidin (Invitrogen) for $20 \mathrm{~min}$ at room temperature. After washing, coverslips were mounted and observed under the fluorescence microscope as described above.

\section{Immunohistochemistry}

The brains were dissected out from adult B6 mice and fixed with $4 \%$ paraformaldehyde and $4 \%$ sucrose in PBS for at least $4 \mathrm{~h}$ at $4^{\circ} \mathrm{C}$. Cryoprotection was performed by incubating brain tissues in gradient sucrose-PBS solution (10\% sucrose for $1 \mathrm{~h}, 20 \%$ sucrose for $1 \mathrm{~h}$, and $30 \%$ sucrose overnight) at $4^{\circ} \mathrm{C}$. Cryosections of $10 \mu \mathrm{m}$ thickness were used for immunohistochemistry. For 3,3'-diaminobenzidine (DAB) staining, the slices were first incubated in $0.6 \%$ hydrogen peroxide for $30 \mathrm{~min}$ to consume endogenous peroxides. Next, the slices were digested with $25 \mu \mathrm{g} / \mathrm{ml}$ trypsin for $5 \mathrm{~min}$ at $37^{\circ} \mathrm{C}$ and heated in microwave in $0.2 \%$ citrate acid buffer, $\mathrm{pH} 6.0$, for $90 \mathrm{~s}$ to enhance antigen accessibility and diminish nonspecific background staining. After cooling down and rinsing with PBS, the sections were permeabilized and blocked by $10 \%$ normal goat serum (NGS) plus $0.2 \%$ Triton $\mathrm{X}-100$ in PBS for $1 \mathrm{~h}$ at room temperature in humidified atmosphere After blocking, the sections were incubated with primary antibodies overnight at $4^{\circ} \mathrm{C}$, followed by washing with PBS three times gently and incubation with biotinylated rabbit secondary antibody for $1 \mathrm{~h}$ at room temperature. Then the slices were washed with PBS and incubated for 30 min with Vectastain ABC reagent (Vector Laboratories, Burlingame, CA). After washing, the slices were developed in DAB peroxidase substrate solution (Vector Laboratories) for 5-10 min, and reactions were stopped by washing with water. For fluorescent staining, after blocking and primary antibody incubation, the slices were directly labeled by fluorescent secondary antibodies at room temperature for $1 \mathrm{~h}$, and then $4^{\prime}, 6^{\prime}$-diamidino-2-phenylindole 
(DAPI) was used to stain the nucleus at room temperature for $15 \mathrm{~min}$. Finally, the slices were dehydrated and mounted with VectaMount solution (Vector Laboratories).

\section{Biotinylation assay}

HEK 293T cells were washed three times with PBS supplemented with $0.5 \mathrm{~mm} \mathrm{CaCl}_{2}$ and 0.5 $\mathrm{mM} \mathrm{MgCl}_{2}$ (B buffer) and treated with 0.5 $\mathrm{mg} / \mathrm{ml}$ sulfo-succinimidyl-6-(biotinamido) hexanoate (sulfo-NHS-LC-biotin; Pierce) in B buffer for $5 \mathrm{~min}$ at room temperature. The free sulfo-NHS-LC-biotin was removed by washing the cells twice with $100 \mathrm{~mm}$ glycine in B buffer followed by two washes with B buffer. The biotinylated cells were solubilized with $1 \mathrm{ml}$ of radioimmunoprecipitation assay buffer $(10 \mathrm{~mm}$ Tris, pH 7.4, 150 mm NaCl, 1 mm EDTA, 0.1\% SDS, $1 \%$ Triton X-100, and $1 \%$ sodium deoxycholate). The samples were centrifuged at $14,000 \mathrm{rpm}$ for $15 \mathrm{~min}$ at $4^{\circ} \mathrm{C}$. A sample of this supernatant was saved for estimation of the total protein. The remaining supernatants were incubated with $50 \mu \mathrm{l}$ of $50 \%$ slurry of NeutrAvidin beads (Pierce) for $1 \mathrm{~h}$ at $4^{\circ} \mathrm{C}$ with constant rotation. After several washes, the biotinylated surface proteins were eluted from the NeutrAvidin beads in $100 \mu$ l of $1 \times$ SDS sample buffer. The samples were subjected to SDS-PAGE and Western blot analysis. The surface GluR2 ratio was calculated by densitometry analysis using a linear standard curve of total GluR2.

\section{Results}

Identification of ICA69 as a major binding partner of PICK1's

\section{BAR domain}

To identify PICK1-interacting protein(s), we performed coimmunoprecipitation from brain tissue using an N-terminal PICK1 antibody. The immunoprecipitation products were resolved by SDS-PAGE and stained with Coomassie blue. One prominent band around $70 \mathrm{kDa}$ was consistently pulled down by the PICK1 antibody (Fig. 1 $A$ ). This band was not seen in the control, in which the PICK1 antibody was blocked by the antigenic peptide. We excised this band and subjected it to mass spectrometry analysis, which identified eight different peptides that belong to a single protein called ICA69 (Fig. 1B).

ICA69 was initially cloned by its reaction with autoantibodies from type- 1 or insulin-dependent diabetes mellitus patients (Pietropaolo et al., 1993). Interestingly, ICA69 also contains a BAR domain, which spans the $\mathrm{N}$-terminal half of the protein (Fig. 1 B, C). The C-terminal half of ICA69 contains a domain with no apparent homology to other known proteins, and we tentatively named it ICAC (ICA69 $\mathrm{C}$ terminus) domain here. Using the yeast two-hybrid assay, we found that the BAR domain of PICK1 was sufficient to interact with ICA69 (Fig. 1C). Similarly, the BAR
A

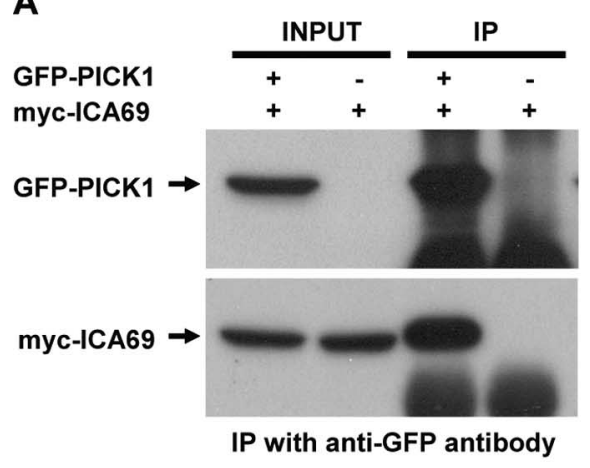

B
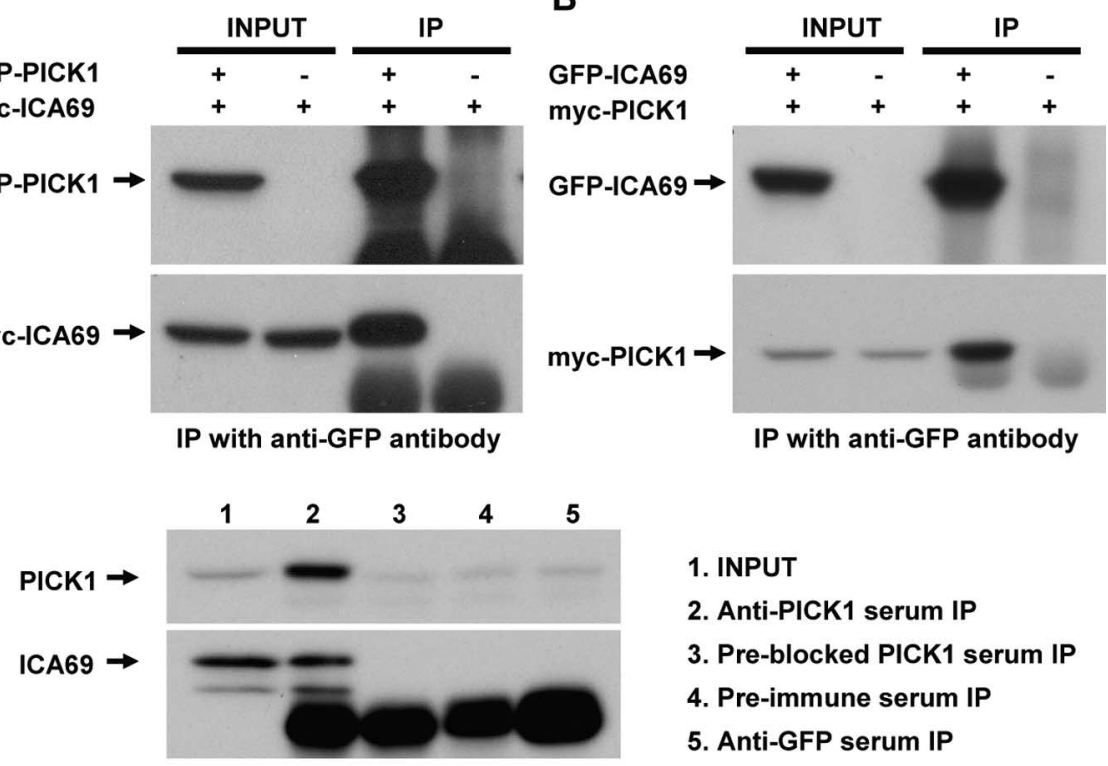

1. INPUT

2. Anti-PICK1 serum IP

3. Pre-blocked PICK1 serum IP

4. Pre-immune serum IP

5. Anti-GFP serum IP

D

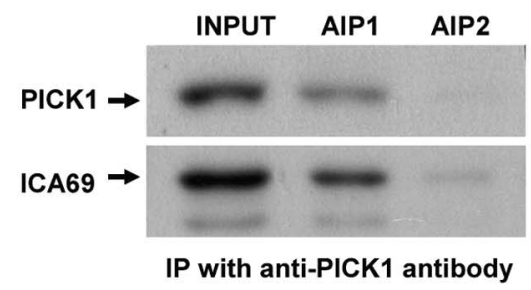

E

$\mathbf{F}$
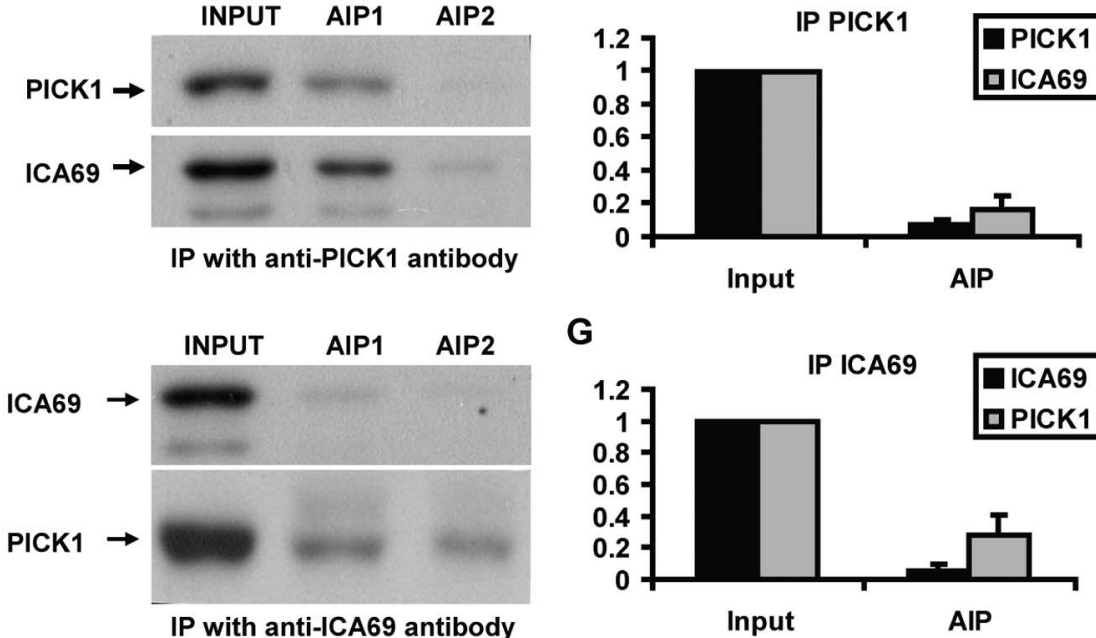

G

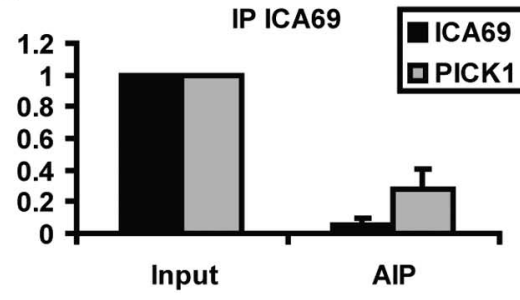

Figure 2. ICA69 and PICK1 form tight complexes in vitro and in vivo. A, Myc-ICA69 was transfected into 293T cells with or without GFP-PICK1. PICK1 was immunoprecipitated using an anti-GFP antibody. Anti-GFP antibody (top) or anti-myc antibody (bottom) was used for Western blotting. Expression of GFP-PICK1 and myc-ICA69 in 293T cells is indicated in the INPUTs, which are the cell lysates used for IP. The IP products indicate that myc-ICA69 was only pulled down in the presence of GFP-PICK1 but not in the control, when myc-ICA69 was expressed alone. B, GFP-ICA69 and myc-PICK1 were transfected into 293T cells and immunoprecipitation was performed as described in $A$. Similarly, myc-PICK1 was only pulled down when ICA69 was present. C, Coimmunoprecipitation from rat brain homogenate was performed using an anti-PICK1 antibody. The antibody pulled down PICK1 itself (top) and a significant amount of ICA69 (bottom), indicating that ICA69 and PICK1 interact with each other in vivo. In the control experiments, when PICK1 antibody was preblocked by the antigenic peptide, or when preimmune serum or unrelated anti-GFP serum was used, both PICK1 and ICA69 were not precipitated. D, PICK1 was consecutively immunoprecipitated with anti-PICK1 antibodies from rat brain homogenate. The rat brain homogenate before immunoprecipitation was designated as INPUT. The rat brain homogenate after the first immunoprecipitation was designated as AIP1. AIP1 was subsequently immunoprecipitated with PICK1 antibodies again, and the rat brain homogenate after the second immunoprecipitation was designated as AIP2. Immunoprecipitation was performed consecutively until almost all of the PICK1 was depleted from the rat brain homogenate, which usually took two to three rounds of immunoprecipitation. Equal volumes of INPUT and AIP samples were loaded, the intensity of INPUT was normalized to 1 , and the intensities of the AIPs were normalized in relation to corresponding INPUT. The last AIP from the consecutive immunoprecipitation was used for the quantification and bar chart. $\boldsymbol{E}$, The quantification data show that when PICK1 is almost completely depleted ( $7.4 \pm 3.2 \%$ in AIP, mean \pm SEM, $n=3$ ), there is $\sim 16.8 \pm 7.6 \%$ of ICA69 left in AIP. This translates to $\sim 90 \%$ of ICA69 being immunoprecipitated if PICK1 was $100 \%$ immunoprecipitated. $F$, Consecutive coimmunoprecipitation was performed as described in $\boldsymbol{D}$ using anti-ICA69 antibodies. $\mathbf{G}$, The quantification data show that when ICA69 was nearly completely depleted ( $5.6 \pm 3.6 \%$ in AIP, mean $\pm \mathrm{SEM}, n=3)$, there is $\sim 28.2 \pm 12.2 \%$ of PICK1 left in AIP. This translates to $76 \%$ of PICK1 associating with ICA69 in rat brain homogenates. 

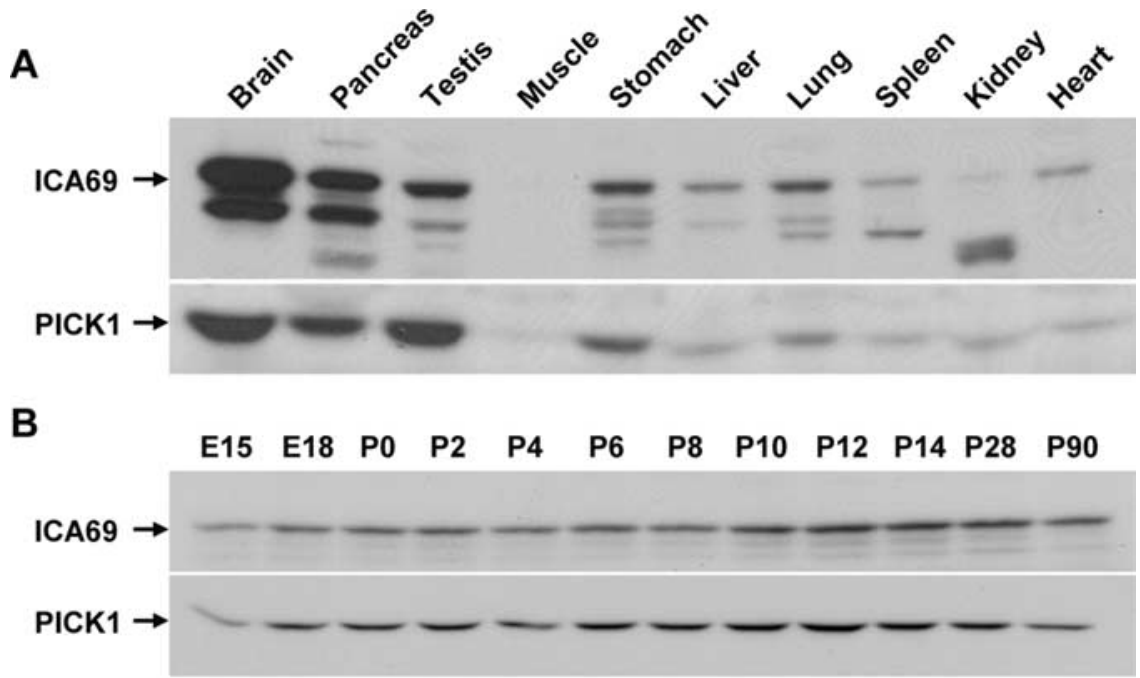

C

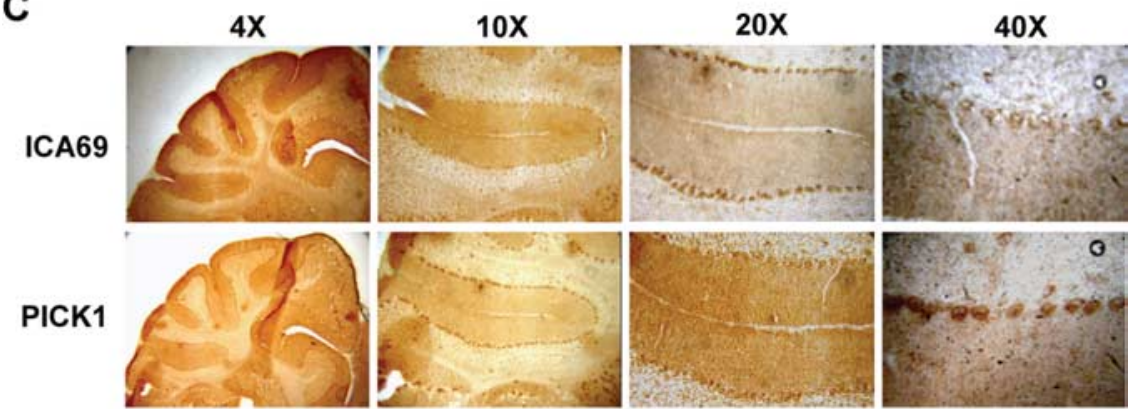

D
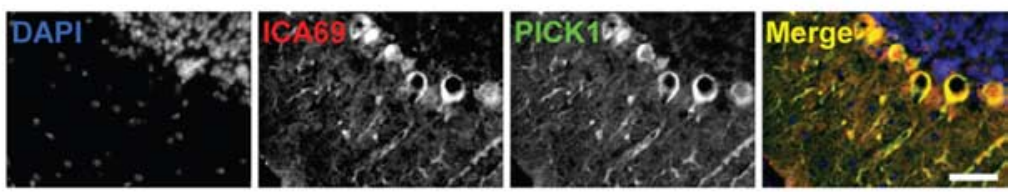

E
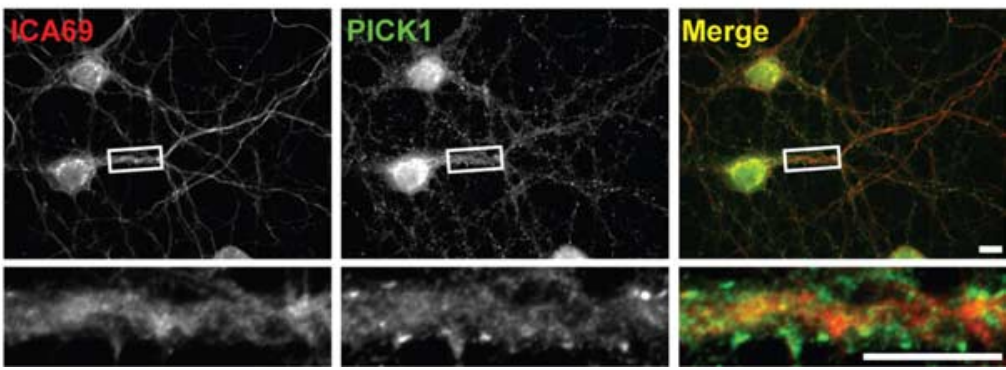

Figure 3. ICA69 colocalizes with PICK1 in most tissues, developmental stages, cells, and subcellular regions, except for synapses. $\boldsymbol{A}$, Different tissues were dissected from rats and homogenized to obtain the total proteins. Equal amounts of proteins were loaded to SDS-PAGE and analyzed by Western blotting. The highest level of both PICK1 and ICA69 were found in the brain, followed by the pancreas and testis. B, Brains were dissected from B6 mice at different developmental stages, from E15 to P90, and homogenized to obtain the total proteins. Equal amounts of proteins were loaded to SDS-PAGE and analyzed by Western blotting. Both PICK1 and ICA69 are expressed from early embryonic stages to adult, and the expressions peak at $\sim 2$ weeks after birth. $C$, Immunohistochemistry studies were performed on mice brain cryosections using anti-PICK1 and anti-ICA69 antibodies. Both proteins are highly expressed in cerebellum, especially at the Purkinje neurons and the molecular layer, which harbors the dendrites of Purkinje neurons. D, Double staining of PICK1 (green) and ICA69 (red) on 10- $\mu \mathrm{m}$-thick brain cryosections, using a guinea pig antibody against PICK1 and a rabbit antibody against ICA69. ICA69 and PICK1 colocalized very well in the perinuclear region and dendrites of Purkinje neurons. The nuclei were stained by DAPI (blue). Scale bar, $10 \mu \mathrm{m}$. $E$, Double staining of PICK1 (green) and ICA69 (red) on cultured hippocampal neurons (day in vitro 17) shows that they colocalized well in the perinuclear region and dendrites. However, ICA69 is notably absent from the synapses, where PICK1 is expressed. Scale bar, $10 \mu \mathrm{m}$.

domain of ICA69 was sufficient to interact with the BAR domain of PICK1. BAR domains form crescent-shaped dimers (Tarricone et al., 2001; Peter et al., 2004). The interaction of the PICK1 and ICA69 BAR domains suggests that PICK1 and ICA69 could each contribute one subunit to form a heteromeric BAR domain complex. Using the yeast two-hybrid assay, we also detected an interaction between the ICAC domain of ICA69 and full-length PICK1. However, we could not map the ICAC domain's binding site on PICK1, because it does not bind to the PDZ, the BAR domain, or the C-terminal region of PICK1 (Fig. 1C).

\section{ICA69 and PICK1 form tight complexes in vitro and in vivo}

To verify the interaction between PICK1 and ICA69, we performed both in vitro and in vivo coimmunoprecipitation experiments. GFP- or myc-tagged PICK1 and ICA69 were transfected into HEK 293T cells and immunoprecipitated with an anti-GFP antibody. As expected, myc-ICA69 was readily coimmunoprecipitated with GFPPICK1 (Fig. 2A). No ICA69 was pulled down in the control experiment, when mycICA69 was expressed in the absence of GFPPICK1 (Fig. 2A). In contrast, when ICA69 was immunoprecipitated, PICK1 was also robustly pulled down with ICA69 (Fig. 2 B). To detect endogenous ICA69 from the in vivo coimmunoprecipitation experiments, we generated a rabbit polyclonal antibody against ICA69. This antibody recognizes a doublet band around $70 \mathrm{kDa}$ from rat brain homogenate, and both bands can be blocked by the antigenic peptide (supplemental Fig. S1, available at www.jneurosci.org as supplemental material). We then performed in vivo coimmunoprecipitation using the antiPICK1 antibody to pull down PICK1 from rat brain homogenate. Again, ICA69 was found to coimmunoprecipitate robustly with PICK1 (Fig. 2C), confirming PICK1's in vivo interaction with ICA69. In the control experiments, ICA69 was not pulled down when PICK1 antibody was preblocked by the antigenic peptide or when preimmune serum or anti-GFP serum were used, indicating the specificity of the coimmunoprecipitation.

To estimate the percentages of PICK1 and ICA69 that were associated with each other in vivo, we performed quantitative coimmunoprecipitation from rat brain homogenates. We first completely depleted PICK1 from the rat brain homogenate by consecutive immunoprecipitation using the anti-PICK1 antibody. We found that when PICK1 was completely depleted from the brain homogenate, most of the ICA69 was also depleted (Fig. 2D). We quantified the result using densitometry and estimated that $\sim 90 \%$ of ICA69 associates with PICK1 in rat brain homogenate (Fig. 2E). Similarly, when ICA69 was completely depleted from the brain homogenate using the ICA69 antibody, the majority of PICK1 was also depleted (Fig. $2 F$ ). Quantification of our immu- 
noblots revealed that $\sim 76 \%$ of PICK 1 associates with ICA69 in the brain (Fig. 2G). These results indicate that the majority of PICK1 and ICA69 form complexes with each other in the brain.

\section{ICA69 colocalizes with PICK1 except at synapses}

ICA69 was initially identified from pancreatic $\beta$ cells and subsequently found to be expressed in many other tissues, including the brain (Pietropaolo et al., 1993; Pilon et al., 2000). PICK1 is known to be highly expressed in the brain, but its expression in the pancreas has not been examined (Xia et al., 1999). To get a more complete picture about the distribution pattern of ICA69 in relationship with PICK1, we compared the expression levels of ICA69 and PICK1 in various tissues by immunoblot analysis. Identical amounts of protein from different tissue homogenates were resolved by SDS-PAGE and immunoblotted with antibodies recognizing either endogenous PICK1 or ICA69. As shown in Figure $3 A$, the expression levels of PICK1 and ICA69 were well correlated in different tissues. The highest expression of both PICK1 and ICA69 was found in the brain. PICK1 and ICA69 were also highly expressed in the pancreas and testis, followed by significant expression in the stomach and lung and modest expression in liver, spleen, heart, and kidney. The lowest expression of both PICK1 and ICA69 was found in skeletal muscles, where they were almost undetectable. In addition, our ICA69 antibody recognizes multiple bands in different tissues. This suggests either alternative splicing of the ICA69 mRNA and/or that different posttranslational modifications occur in ICA69 in different tissues. We also examined the developmental profiles of ICA69 and PICK1 in mouse brains. Equal amounts of protein from different developmental stages of mouse brains were used for SDS-PAGE and immunoblot analysis. As shown in Figure 3B, ICA69 and PICK1 have very similar developmental profiles. They are both expressed as early as embryonic day 15. The expressions of both proteins gradually increase, reach their peaks at $\sim 2$ weeks after birth, and remain expressed in adult mice brains.

The localization of PICK1 and ICA69 in different regions and cell types of the brain were also examined using immunohistochemical analysis. PICK1 and ICA69 were found to be coexpressed in many brain regions. The highest expression levels of both PICK1 and ICA69 were in the cerebellar Purkinje neurons (Fig. 3C,D). The molecular layer of the cerebellar cortex, which harbors the dendrites of Purkinje neurons, was also heavily stained. High-magnification photos indicate that both PICK1 and ICA69 were located in the perinuclear region and dendrites of Purkinje neurons (Fig. 3D). To examine the detailed subcellular localization
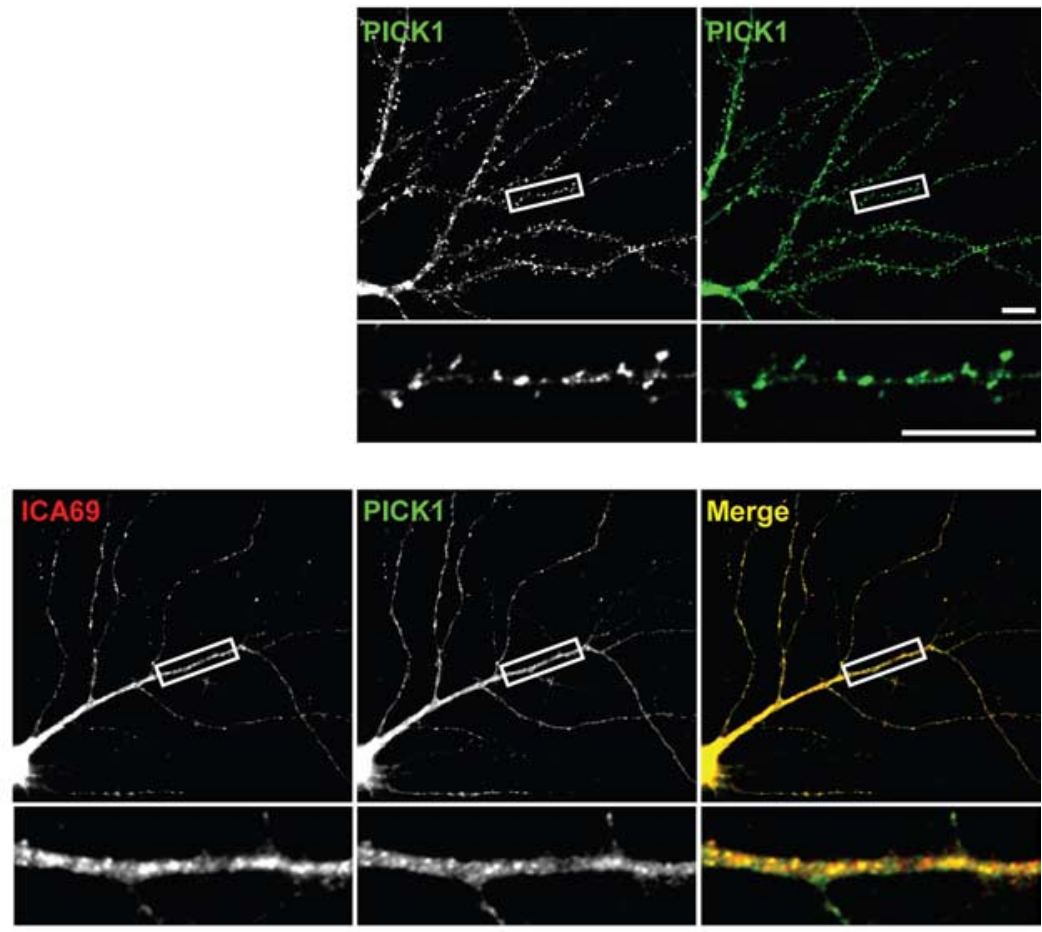

PICK1 cluster number

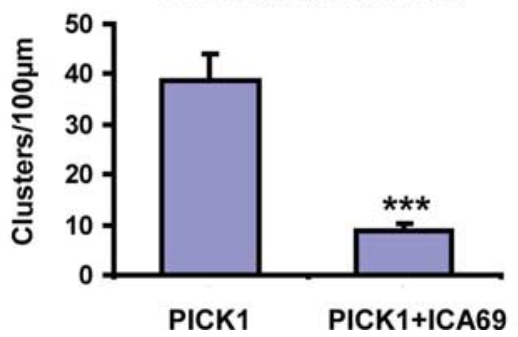

PICK1 cluster intensity

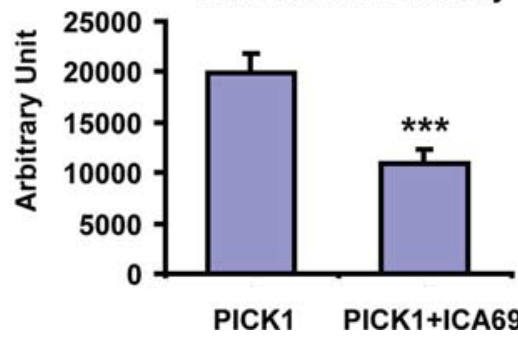

Figure 4. ICA69 redistributes PICK1 from synapses to dendrites. $\boldsymbol{A}$, Cultured hippocampal neurons were transfected with myc-PICK1 and stained with a guinea pig anti-PICK1 antibody. PICK1 (green) was found to be highly clustered along the dendrites transfected with both myc-PICK1 and myc-ICA69 and double stained with a guinea pig anti-PICK1 antibody and a rabbit antilusters significantly decreased in the presence of ICA69 (PICK1 cluster number per $100 \mu \mathrm{m}$ : PICK1 $=38.9 \pm 5.0$ PICK1 + ICA69 = 8.8 $\pm 1.4 ;$ PICK1 cluster intensity: PICK1 = 19,918.3 \pm 1931.1, PICK1 + ICA69 = 10,843.7 \pm 1511.1, mean \pm SEM; $n=26 ;{ }^{* * *} p<0.001$, Student's $t$ test). Error bars represent SEM.

of PICK1 and ICA69 in neurons, we stained cultured hippocampal neurons with antibodies against PICK1 and ICA69. Although PICK1 and ICA69 colocalized well with each other in the dendrites and the perinuclear regions of the cell bodies, ICA69 was surprisingly missing at synapses, where PICK1 is enriched (Fig. $3 E$ ). This suggests that the heteromeric BAR domain complexes of PICK1 and ICA69 are mainly located in cell bodies and dendrites. At synapses, PICK1 is likely to exist as a homomeric BAR domain complex or in complex with other proteins.

\section{ICA69 inhibits synaptic targeting of PICK1}

The absence of ICA69 protein at synapses raises the possibility that ICA69 may form complexes with PICK1 and prevent PICK1 from targeting to synapses. To test this possibility, we transfected ICA69 together with PICK1 into cultured hippocampal neurons. 
A
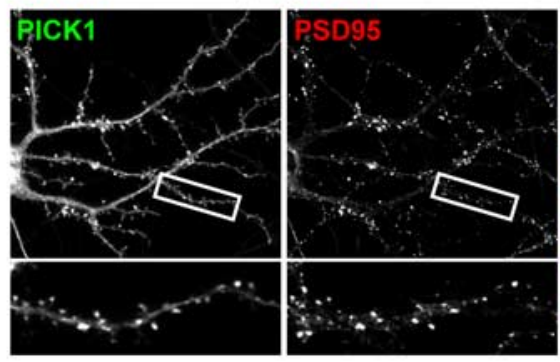

B
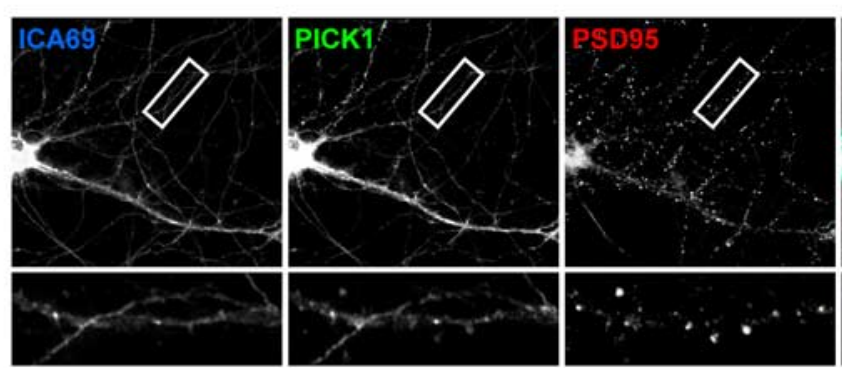

C
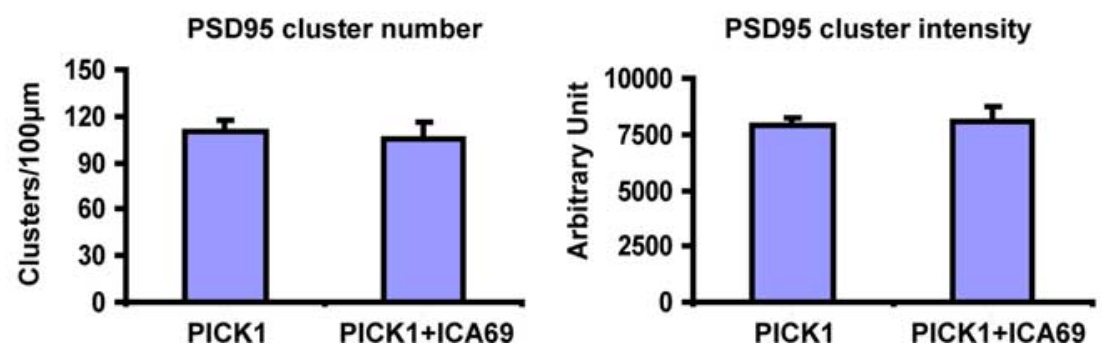

Figure 5. Overexpression of ICA69 does not affect PSD-95 distribution. $A$, Cultured hippocampal neurons were transfected with myc-PICK1 and double stained with a guinea pig anti-PICK1 antibody and a mouse anti-PSD-95 antibody. PICK1 (green) colocalized with PSD-95 (red) at the excitatory synapses along the dendrites. Scale bar, $10 \mu \mathrm{m}$. B, In neurons transfected with both PICK1 and ICA69 (blue), the amount of PICK1 was significantly reduced from the synaptic region and no longer colocalized with PSD-95 clusters. Instead, PICK1 colocalized with ICA69 inside the dendrites. However, the PSD-95 clusters along the dendrites were not significantly different from the neurons without ICA69 in $\boldsymbol{A}$. C, Quantification data of $\boldsymbol{A}$ and $\boldsymbol{B}$. Neither the number nor the intensity of synaptic PSD-95 clusters changed significantly in the presence of ICA69 (PSD-95 cluster number per $100 \mu \mathrm{m}$ : PICK1 $=109.9 \pm$ 7.2, PICK1 + ICA69 = 105.8 \pm 10.5; PSD-95 cluster intensity: PICK1 = 7885.1 \pm 317.9 , PICK1 + ICA69 = 8098.7 \pm 654.3 , mean $\pm \mathrm{SEM} ; n=13 ; p>0.05$, Student's $t$ test). Error bars represent SEM.

Without the transfected ICA69, PICK1 formed many synaptic clusters along the dendrites (Fig. 4A). These clusters were synaptic, because they colocalized with postsynaptic markers such as PSD-95 (Fig. 5). When ICA69 was transfected with PICK1, there were almost no synaptic clusters of PICK1 (Fig. 4 B). Meanwhile, patches of PICK1 and ICA69 were found along the dendritic shafts. Quantification of these data indicated that both the number and intensity of PICK1 synaptic clusters were significantly reduced in the presence of ICA69 (Fig. 4C). These results suggest that ICA69 indeed redistributes PICK1 from synapses to dendritic shafts. To make sure that ICA69's effect on PICK1 is specific, we expressed ICA69 together with GFP-tagged PSD-95, a well studied synaptic PDZ domain-containing protein. As shown in supplemental Fig. S2, expression of ICA69 in neurons did not significantly alter the number or intensity of GFP-PSD-95 clusters.

To rule out the possibility that the disappearance of PICK1 from synapses was a secondary effect of losing spines or excitatory synapses, we stained PICK1 together with endogenous PSD-95,
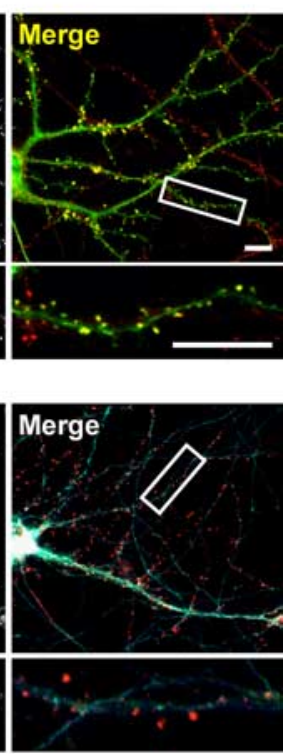

which serves as a marker for excitatory synapses. As shown in Figure 5A, without overexpression of ICA69, PICK1 colocalized with PSD-95 at the excitatory synapses. In the presence of ICA69, there was largely no PICK1 at the synapses, whereas PSD-95 clusters were still present (Fig. 5B). We quantified these results and found that ICA69 did not alter either the number or the intensity of the PSD-95 clusters (Fig. 5C), indicating that ICA69 does not reduce excitatory synapses. To make sure there is no change in spine morphology, we used rhodamine-phalloidin to label F-actin, a cytoskeleton protein enriched in spines, to mark spines. We did not find any notable change in the spine morphology as revealed by rhodamine-phalloidin. Neither the cluster number nor the intensity of rhodamine-phalloidin-labeled spines was affected by overexpression of ICA69 (supplemental Fig. S3). These results indicate that ICA69 dose not alter the structure of spines.

\section{ICA69 regulates PICK1-mediated trafficking of GluR2}

PICK1 has been reported to interact with GluR2 and induce clustering of GluR2 (Dev et al., 1999; Xia et al., 1999). The clustering of GluR2 by PICK1 requires the lipid-binding capability of PICK1's BAR domain (Jin et al., 2006). Because ICA69 forms a heteromeric BAR domain complex with PICK1, it raises the possibility that ICA69 may regulate PICK1-induced clustering of GluR2. To test this, we overexpressed ICA69 together with PICK1 and GluR2 in 293T cells. When GluR2 was coexpressed with PICK1, they formed numerous coclusters in the cytosol of 293T cells (Fig. 6A). Interestingly, when ICA69 was expressed together with PICK1 and GluR2, the PICK1-GluR2 coclusters completely disappeared (Fig. 6B). Without PICK1, both ICA69 and GluR2 were diffused in the 293T cells (Fig. 6C), similar to the patterns when ICA69 and GluR2 were expressed alone (Fig. 6D). This suggests that ICA69 indeed regulates PICK1-mediated AMPA receptor trafficking.

In view of the results that ICA69 disrupts PICK1-GluR2 coclustering in 293T cells and that ICA69 inhibits synaptic targeting of PICK1 in neurons, we wondered whether ICA69 could also affect synaptic targeting of GluR2 in neurons. To test this hypothesis, we overexpressed ICA69 in cultured hippocampal neurons together with PICK1 and GluR2. We found that when ICA69 was absent, PICK1 colocalized with GluR2 at the synapses (Fig. 7A). Overexpression of ICA69 redistributed PICK1 to the dendrites and also significantly reduced the number of GluR2 clusters at the synapses (Fig. 7B). Quantification of these results indicated that ICA69 reduced the number of GluR2 clusters by $\sim 40 \%$ (Fig. $7 C$ ).

Because PICK1 has been reported to regulate surface ex- 
pression of AMPA receptors (Jin et al., 2006), we further tested whether ICA69 plays any role in AMPAR surface expression. We used biotinylation assay to measure surface expression of GluR2 in HEK 293T cells transfected with GluR2 alone, GluR2 with PICK1, or GluR2 with PICK1 and ICA69. Biotin-labeled surface proteins were pulled down by NeutrAvidin beads, and surface GluR2 was detected by rabbit anti-GluR2/3 antibody. Similar to what we observed previously (Jin et al., 2006), PICK1 significantly reduced the surface level of GluR2, compared with cells transfected with GluR2 alone (Fig. 8 A, top and middle). When ICA69 was added in, surface GluR2 was further decreased (Fig. $8 \mathrm{~A}$, bottom). Quantification showed that surface GluR2 was reduced by $>50 \%$ in the presence of PICK1 and ICA69 (Fig. $8 \mathrm{~B})$. We also performed surface staining of GluR2 on cultured hippocampal neurons. In HA-GluR2- and GFP-PICK1cotransfected neurons, PICK1 targeted to synapse and surface GluR2 was detected on the spine (Fig. $8 C$ ). With overexpressed ICA69, PICK1 was absent from synapses, and surface GluR2 at synapses also dramatically decreased (Fig. $8 D)$. We also noticed notable decrease of surface GluR2 on dendrites and cell bodies of ICA69-transfected neurons, indicating that ICA69's role on surface expression of GluR2 is not limited to synapses. Quantification showed that overexpression of ICA69 significantly reduced surface GluR2 level on both dendrites and synapses (Fig. $8 E$ ). We also used RNAi to knock down ICA69, but, unexpectedly, knocking down ICA69 caused a dramatic reduction of total GluR2, which prevented us from evaluating the effect of ICA69 downregulation on surface expression of GluR2 (M. Cao and J. Xia, unpublished data).

ICA69 regulates AMPA receptor trafficking by preventing formation of homomeric PICK1 BAR domain complexes

We then sought to find out how ICA69 prevents synaptic targeting of PICK1 and trafficking of AMPA receptors. Because the BAR domain of PICK1 was found to interact directly with lipid molecules and this interaction is important for PICK1's synaptic targeting and AMPA receptor trafficking (Jin et al., 2006), we wondered whether the BAR domain of ICA69 could also bind to lipids. This was tested using a liposome sedimentation assay. MBP-tagged full-length ICA69, BAR domain (ICA-BAR) and the C-terminal domain of ICA69 (ICAC) fusion proteins were incubated with liposomes and subjected to ultracentrifugation. Fusion proteins that bound to liposomes would be cosedimented with liposomes and detected in the pellet fraction after centrifugation. We found that ICA69 was fully capable of binding to lipids, as it readily came down with liposomes (Fig. 9A). As expected, the BAR domain of ICA69 bound to liposomes but the ICAC domain did not, indicating that the BAR domain of ICA69 is responsible for lipid binding (Fig. 9A).

Because PICK1-mediated clustering and synaptic targeting
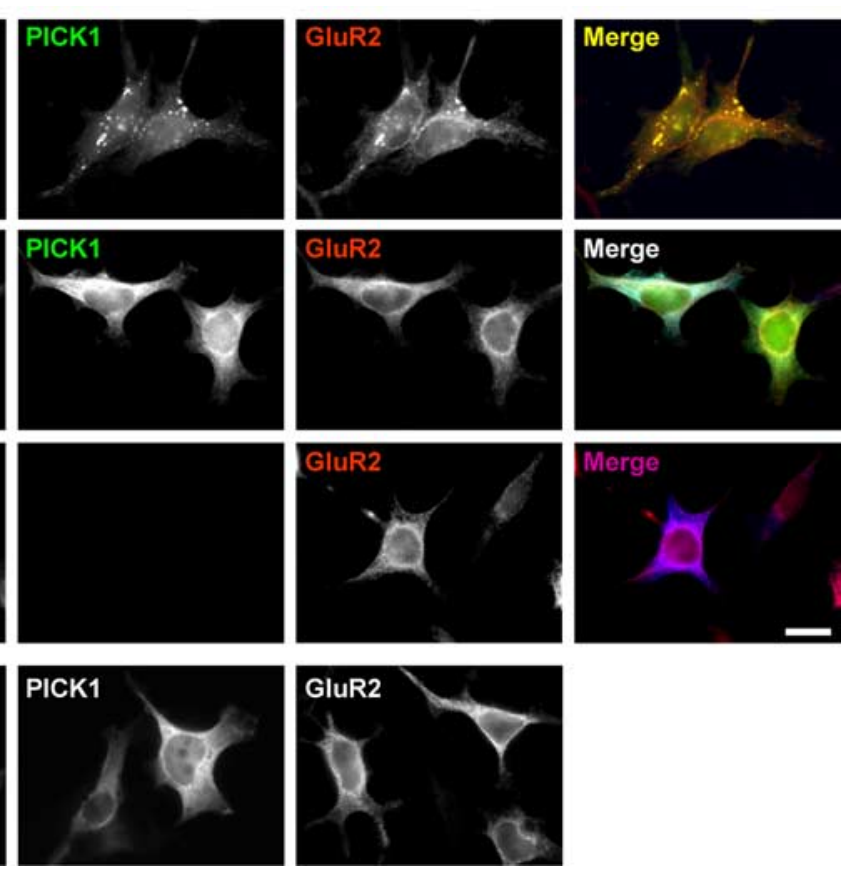

Figure 6. Overexpression of ICA69 disrupts PICK1-GluR2 coclusters in 293T cells. HEK 293T cells were transfected with myc( GluR2, these clusters completely disappeared. C, When ICA69 was transfected with GluR2 without PICK1, both ICA69 and GluR2 were diffuse in the cytosol. D, When ICA69, PICK1, and GluR2 were expressed alone, they were all diffusely localized in the cytosol.

of GluR2 requires lipid binding of PICK1's BAR domain (Jin et al., 2006), we wondered whether ICA69 could prevent the synaptic targeting of PICK1 and AMPA receptors by inhibiting the lipid binding of PICK1. To test this possibility, we measured the lipid-binding capability of PICK1 in the presence or absence of ICA69, using the liposome sedimentation assay described above. As shown in Figure 9B, the amount of PICK1 associated with the liposomes did not change significantly in the presence of ICA69. This result suggests that ICA69's effects on PICK1 and AMPA receptor trafficking are unlikely through alteration of PICK1's lipid-binding capability.

We then examined whether ICA69 could affect PICK1mediated AMPA receptor trafficking by directly interacting with GluR2. We cotransfected GluR2 with myc-ICA69 or myc-PICK1 into $293 \mathrm{~T}$ cells. The cell lysates were immunoprecipitated with an anti-myc antibody and immunoblotted to detect the presence of ICA69, PICK1, and GluR2. Whereas GluR2 readily associated with PICK1, as previously reported (Xia et al., 1999), we did not observe any coimmunoprecipitation of ICA69 with GluR2 (Fig. 9C). In addition, we did not notice any change in GluR2 localization when GluR2 was coexpressed with ICA69 in 293T cells in the absence of PICK1 (Fig. 6C). These results indicate that ICA69 does not interact with GluR2 directly and thus does not regulate the cellular distribution of GluR2 in the absence of PICK1.

We then tested whether ICA69 could disrupt PICK1mediated AMPA receptor trafficking by perturbing the interaction between PICK1 and GluR2. GFP-PICK1 and GluR2 were transfected into $293 \mathrm{~T}$ cells with or without ICA69. PICK1 was then immunoprecipitated with an anti-GFP antibody. GluR2 was found to coimmunoprecipitate with PICK1 regardless of the presence of ICA69 (Fig. 9D). This result indicates that ICA69 does not perturb the interaction between GluR2 and PICK1. 
A
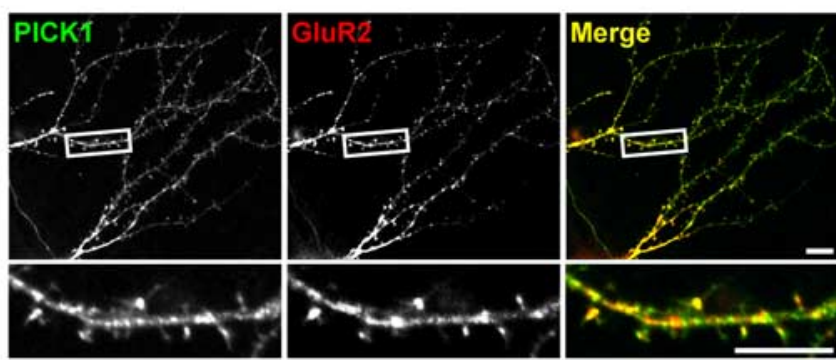

B
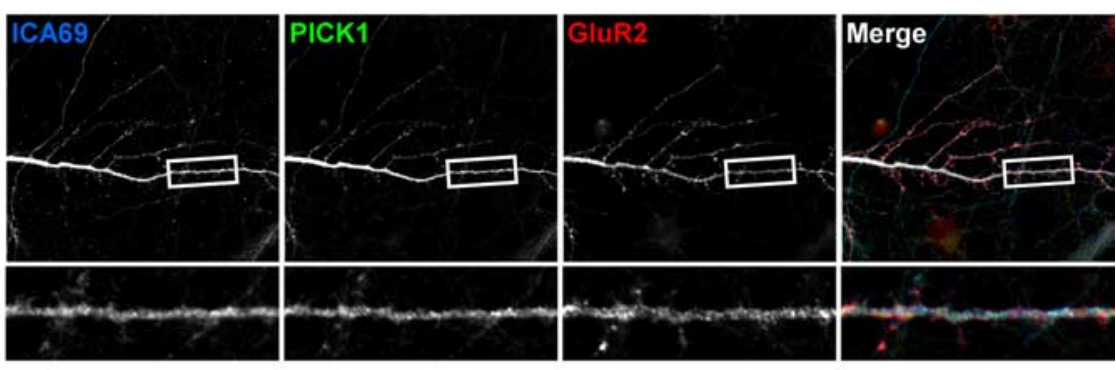

C
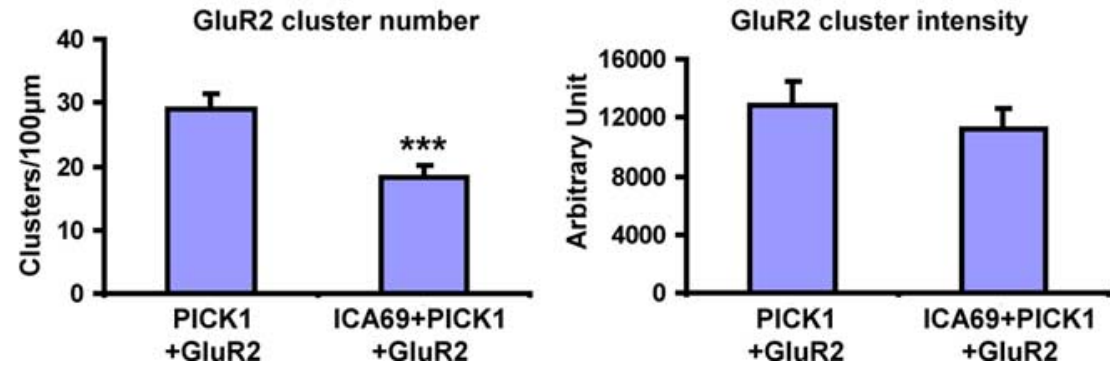

Figure 7. ICA69 inhibits synaptic targeting of GluR2. A, Cultured hippocampal neurons were transfected with myc-PICK1 and HA-GluR2 and double stained with a guinea pig anti-PICK1 antibody and a mouse anti-HA antibody. PICK1 (green) and GluR2 (red) colocalized at synapses along the dendrites. Scale bar, $10 \mu \mathrm{m}$. $\boldsymbol{B}$, When ICA69 was expressed in neurons, ICA69 (blue) not only redistributed PICK1 to the dendrites, but also significantly reduced synaptic targeting of GluR2. C, Quantification data of $\boldsymbol{A}$ and $\boldsymbol{B}$. ICA69 decreased the number of synaptic GluR2 significantly. ICA69 also slightly decreased the intensity of GluR2, but the decrease was not significant (GluR2 cluster number per $100 \mu \mathrm{m}$ : PICK1+GluR2 $=29.0 \pm 2.3$, PICK1+ICA69+GluR2 = 18.1 \pm 1.9 ; GluR2 cluster intensity: PICK1 + GluR2 = 12,830.4 \pm 1608.3, PICK1 + ICA69 + GluR2 = 11,245.4 \pm 1416.5 , mean \pm SEM; $n=$ $106 ;{ }^{* * *} p<0.001$, Student's $t$ test). Error bars represent SEM.

Finally, we tested whether ICA69 could alter the capability of PICK1 to self-associate, because dimerization of PICK1 is required for its clustering of GluR2. PICK1, with different tags (GFP or myc), was transfected into 293T cells with or without myc-ICA69. GFP-PICK1 was immunoprecipitated with an antiGFP antibody. As expected, myc-PICK1 coprecipitated with GFPPICK1 in the absence of ICA69 (Fig. 9E), confirming the capability of PICK1 to form homodimers. However, in the presence of ICA69, the amount of myc-PICK1 that coprecipitated with GFP-PICK1 was significantly reduced (Fig. 9E). On the other hand, a large amount of ICA69 was pulled down by GFP-PICK1 and detected by the same anti-myc antibody (Fig. 9E). These results indicate that the heteromeric BAR domain interaction of ICA69 with PICK1 is stronger than PICK1 self-association. ICA69 can prevent the formation of homomeric PICK1 BAR domain complexes and, consequently, PICK1-mediated trafficking of AMPA receptors.

\section{Discussion}

In this study, we identified ICA69 as a major binding partner of PICK1. Interestingly, both ICA69 and PICK1 contain BAR domains and are capable of forming heteromeric BAR domain complexes with each other. The interaction between ICA69 and PICK1 is very robust. In brain, over three-fourths of ICA69 and PICK1 form heteromeric complexes with each other. Not surprisingly, ICA69 coexpresses with PICK1 in many tissues, including the brain, pancreas, and testis. ICA69 and PICK1 also colocalize well in different cells and subcellular regions, including the perinuclear region and dendrites of neurons. One exception is that ICA69 is absent from synapses, suggesting that PICK1 at synapses is likely in homomeric PICK1 BAR domain complexes or in complexes with proteins other than ICA69. This notion is supported by the finding that overexpressed ICA69 redistributes PICK1 from synapses to dendritic shafts, as greater amounts of ICA69 will prevent the formation of homomeric PICK1 BAR domain complexes. We also found that ICA69 regulates PICK1-mediated AMPA receptor trafficking. When coexpressed with PICK1 in HEK 293T cells, ICA69 completely disrupts PICK1induced clustering of GluR2 and decreases the amount of GluR2 on the cell surface. In neurons, ICA69 reduces surface expression and synaptic targeting of GluR2 by preventing formation of homomeric PICK1 complexes. Together, these results suggest that ICA69-PICK1 heteromeric BAR domain complexes maintain a pool of intracellular AMPA receptor at dendrites, while PICK1 homomeric complexes regulate AMPA receptor trafficking at synapses (Fig. 9F).

ICA69 was initially cloned as an autoantigen for type-1 or insulindependent diabetes mellitus (Pietropaolo et al., 1993). It was later discovered that ICA69 was also an autoantigen in primary Sjogren's syndrome, an autoimmune disease affecting the salivary and lacrimal glands (Winer et al., 2002b). ICA69 knock-out mice are grossly normal but have delayed or reduced rates of diabetes and primary Sjogren's syndrome (Winer et al., 2002a,b). The cellular function of ICA69 is not clear. It has been suggested that ICA69 might be involved in neuron-endocrine secretion, as the mutation of ric-19, the Caenorhabditis elegans homolog of ICA69, leads to resistance to inhibitors of acetylcholinesterase, a phenomenon attributed to defective acetylcholine release (Pilon et al., 2000). ICA69 was found to be located around the Golgi and nearby vesicles (Spitzenberger et al., 2003). Our finding that the BAR domain of ICA69 binds to liposomes supports the notion that ICA69 may target to trafficking vesicles. The interaction of ICA69 with PICK1, whose PDZ domain interacts with a number of membrane proteins, suggests that ICA69 may regulate protein trafficking, perhaps using its BAR domain to sense or form trafficking vesicles.

The complexes of PICK1 and ICA69 are likely to have more functions than just regulating AMPA receptor trafficking. In 
addition to AMPA receptors, the PDZ domain of PICK1 also interacts with a larger number of other molecules, mainly membrane proteins ( $\mathrm{Xu}$ and $\mathrm{Xia}$, 2007). It is likely that the heteromeric BAR domain complexes of ICA69 and PICK1 are also involved in regulating the trafficking of these proteins. It would also be interesting to examine what the ICA69 and PICK1 complexes do in nonneuronal cells, for example, in pancreatic $\beta$ cells or salivary gland cells, and if the ICA69 and PICK1 complexes play any roles in the pathogenesis of diabetes or primary Sjogren's syndrome.

We have previously reported that PICK1's BAR domain binds to lipids and that its lipid binding is critical for AMPA receptor trafficking and synaptic plasticity (Jin et al., 2006). The identification of ICA69 and PICK1 heteromeric BAR domain complexes and their functions in AMPA receptor trafficking provides further evidence supporting the role of BAR domains and lipid binding in regulating AMPA receptor trafficking. Our results suggest that there are two pools of AMPA receptors. One is tethered with the heteromeric complexes of ICA69 and PICK1 in the cell bodies and dendrites. The other pool is clustered by homomeric PICK1 complexes at the synapses. It is conceivable that these two pools of receptors can interchange, by changing the preference of PICK1 to form heteromeric or homomeric BAR domain complexes. By regulating the amount of AMPA receptors at synapses, ICA69 and PICK1 could affect the strength of synaptic transmission and therefore mediate synaptic plasticity. It has been reported that the BAR domain of PICK1 can interact with GRIP/ABP and regulate AMPA receptor trafficking (Lu and Ziff, 2005). We did not identify GRIP/ABP in our screening of PICK1 interacting proteins, suggesting that the interaction of GRIP/ABP with PICK1 is probably weaker than that of ICA69 with PICK1. However, it is possible that the interaction strength can be regulated under different conditions and thus the binding of PICK1 to GRIP/ABP and ICA69 may provide flexibility to regulate AMPA receptor trafficking in response to different stimuli that are often present in neurons and that are important to synaptic plasticity.

\section{References}

Barry MF, Ziff EB (2002) Receptor trafficking and the plasticity of excitatory synapses. Curr Opin Neurobiol 12:279-286.

Bredt DS, Nicoll RA (2003) AMPA receptor trafficking at excitatory synapses. Neuron 40:361-379.

Chung HJ, Xia J, Scannevin RH, Zhang X, Huganir RL (2000) Phosphorylation

E
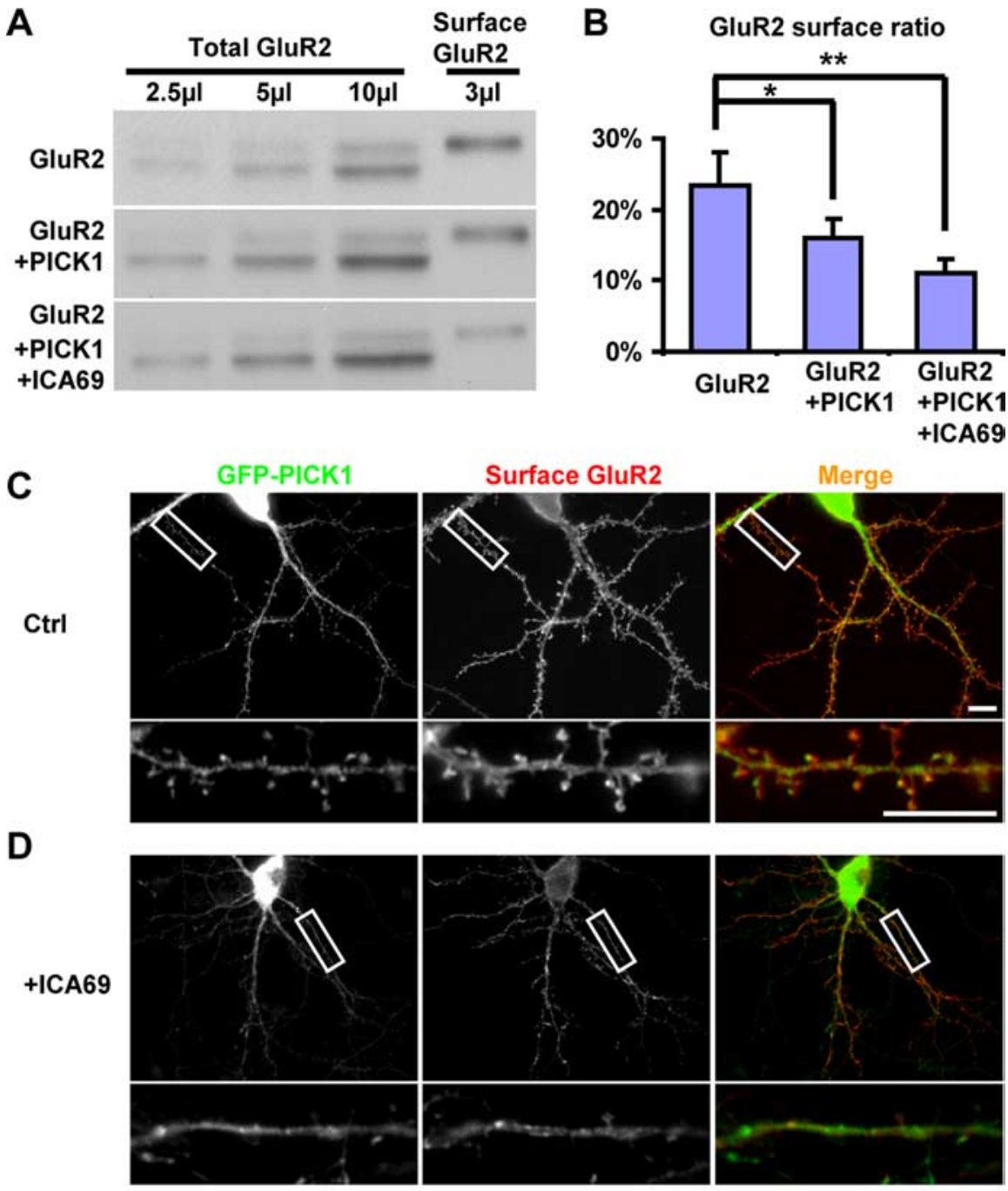

GluR2 dendritic surface intensity

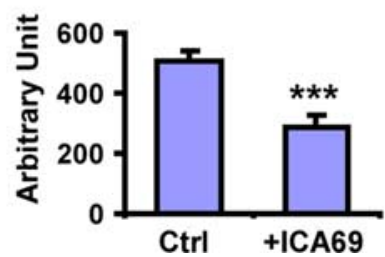

\section{GluR2 synaptic surface intensity}

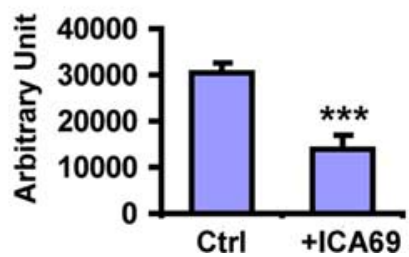

Figure 8. ICA69 reduces surface expression of GluR2. A, HEK 293T cells were transfected with myc-ICA69, GFP-PICK1, and GluR2 in different combinations as indicated. Surface GluR2 was isolated by biotinylation assay and detected by rabbit anti-GluR2/3 antibody. Different amounts of total GluR2 were loaded to obtain a standard curve. Surface GluR2 is notably lower in ICA69transfected cells. $\boldsymbol{B}$, Quantification data of $\boldsymbol{A}$. Surface GluR2 ratio was calculated by fitting the surface samples to the standard curve. Surface GluR2 was significantly less in the presence of PICK1 and further reduced by addition of ICA69 (GluR2 surface ratio: GluR2 alone $=23.3 \pm$ 4.7\%,GluR2 + PICK1 = 16.0 $\pm 2.6 \%$, GluR2 + PICK1 + ICA69 $=11.1 \pm 2.0 \%$, mean \pm SEM, $n=12,{ }^{*} p<0.05,{ }^{* *} p<0.01$, Student's $t$ test). Error bars represent SEM. C, Cultured hippocampal neurons were transfected with HA-GluR2 and GFP-PICK1. Surface GluR2 was stained with a mouse anti-HA antibody before permeabilization. PICK1 (green) and surface GluR2 (red) colocalize at synapses. Scale bar, 10 $\mu \mathrm{m}$. D, When myc-ICA69 was coexpressed with HA-GluR2 and GFP-PICK1, surface expression of GluR2 was dramatically decreased. $\boldsymbol{E}$, Quantification data of $\boldsymbol{C}$ and $\boldsymbol{D}$. Overexpression of ICA69 significantly reduced surface GluR2 in both dendrites and synapses (intensity of surface GluR2 at dendrites: PICK1+GluR2 = 506.9 \pm 33.5 , PICK1 + ICA69+GluR2 = $284.4 \pm 45.6$; intensity of surface GluR2 on synapses: PICK1+GluR2 = 30,331.2 \pm 2410.1 , PICK1+ICA69+GluR2 = 13,889.1 \pm 2957.6 , mean \pm SEM; $n=15 ;{ }^{* *} p<0.01$, ${ }^{* * *} p<0.001$, Student's $t$ test). Error bars represent SEM.

of the AMPA receptor subunit GluR2 differentially regulates its interaction with PDZ domain-containing proteins. J Neurosci 20:7258-7267.

Collingridge GL, Isaac JT, Wang YT (2004) Receptor trafficking and synaptic plasticity. Nat Rev Neurosci 5:952-962. 
A

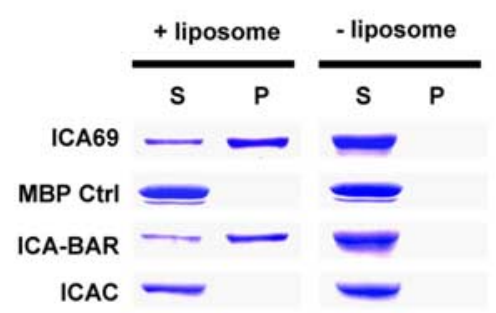

B

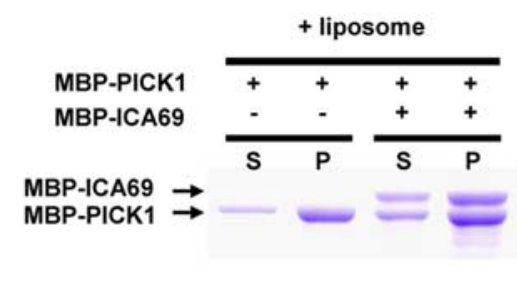

C

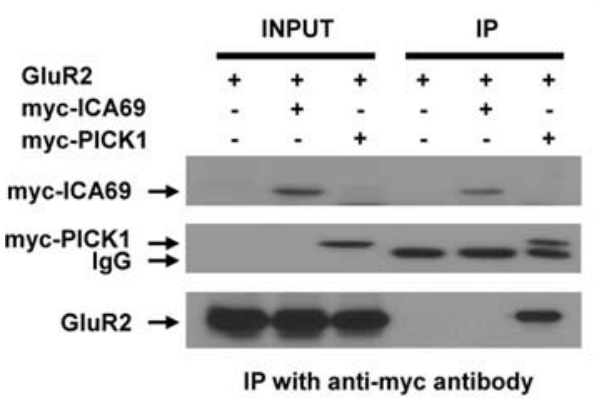

D

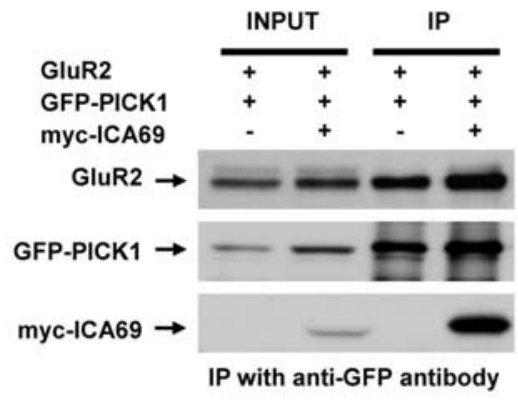

E

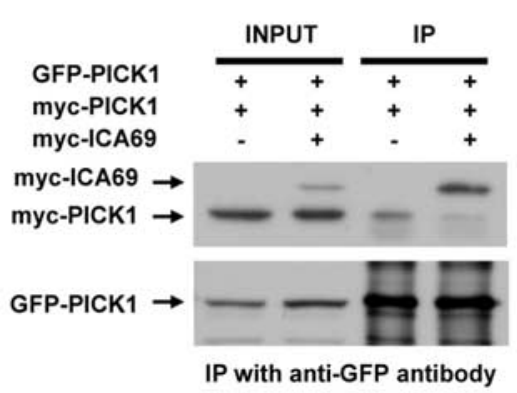

$\mathbf{F}$

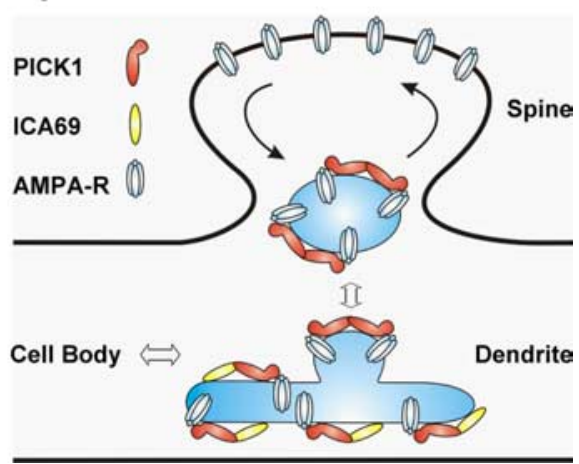

Figure 9. ICA69 reduces formation of homomeric PICK1 BAR domain complexes. A, MBP-tagged ICA69 fusion proteins were mixed with liposomes and subjected to high-speed centrifugation. After centrifugation, substantial amounts of ICA69 were found to come down with liposomes and showed up in the pellet (first panel, left two lanes; $S$, supernatant; $P$, pellet.). Without liposomes, ICA69 was not found in the pellet (first panel, right two lanes). As a control, MBP itself was not found in the liposome-containing pellet (second panel). The BAR domain (ICA-BAR) shows a similar lipid-binding capability as full-length ICA69 (third panel), whereas the (-terminal domain (ICAC) does not bind to lipid (fourth panel). B, ICA69 does not change PICK1's lipid-binding capability. MBP-PICK1 was used for the liposome sedimentation assay with or without MBP-ICA69. Equal amounts of pellet and supernatant were loaded and resolved by SDS-PAGE and stained by Coomassie blue. The level of PICK1 in the liposome-associated pellet fraction is similar with or without ICA69. MBP-ICA69 itself also binds well to liposomes in the presence of PICK1. C, ICA69 does not bind to GluR2 directly. GluR2, myc-PICK1, and myc-ICA69 were expressed in 293T cells as indicated and immunoprecipitated by an anti-myc antibody. GluR2 was only pulled down together with myc-PICK1 but not with myc-ICA69. The cell lysates used for the coimmunoprecipitation were loaded as INPUT to show comparable expression levels of proteins in each experiment. D, ICA69 does not reduce PICK1-GluR2 interaction. GFP-PICK1 and GluR2 were transfected into 293T cells with or without myc-ICA69, and then GFP-PICK1 was immunoprecipitated down with an anti-GFP antibody. The level of GluR2 coimmunoprecipitated with PICK 1 is not reduced in the presence of ICA69, whereas ICA69 itself was robustly coimmunoprecipitated with PICK1 and GluR2 (bottom). E, ICA69 disrupts PICK1 self-association. GFP-PICK1 and mycPICK1 were transfected into 293T cells with or without myc-ICA69. GFP-PICK1 was immunoprecipitated using an anti-GFP antibody. The level of coimmunoprecipitated myc-PICK1 with GFP-PICK1 was significantly reduced in the presence of ICA69. In contrast, ICA69 was robustly coimmunoprecipitated with GFP-PICK1, indicating that ICA69 forms heteromeric BAR domain complexes at the expense of homomeric PICK1 BAR domain complexes. $\boldsymbol{F}$, This model illustrates the role of ICA69 and PICK1 in synaptic targeting of GluR2. There are two pools of GluR2-containing AMPA receptors. The heteromeric BAR domain complexes of ICA69 and PICK1 maintain a pool of AMPA receptors at the dendrites and cell bodies. The homomeric BAR domain complexes of PICK1 maintain a pool of AMPA receptors at the synapses.

Dawson JC, Legg JA, Machesky LM (2006) Bar domain proteins: a role in tubulation, scission and actin assembly in clathrin-mediated endocytosis. Trends Cell Biol 16:493-498.

Dev KK, Nishimune A, Henley JM, Nakanishi S (1999) The protein kinase C alpha binding protein PICK1 interacts with short but not long form alternative splice variants of AMPA receptor subunits. Neuropharmacology 38:635-644.

Gardner SM, Takamiya K, Xia J, Suh JG, Johnson R, Yu S, Huganir RL (2005) Calcium-permeable AMPA receptor plasticity is mediated by subunitspecific interactions with PICK1 and NSF. Neuron 45:903-915.

Henley JM (2003) Proteins interactions implicated in AMPA receptor trafficking: a clear destination and an improving route map. Neurosci Res 45:243-254.

Jin W, Ge WP, Xu J, Cao M, Peng L, Yung W, Liao D, Duan S, Zhang M, Xia J (2006) Lipid binding regulates synaptic targeting of PICK1, AMPA receptor trafficking, and synaptic plasticity. J Neurosci 26:2380-2390.

Kim CH, Chung HJ, Lee HK, Huganir RL (2001) Interaction of the AMPA receptor subunit GluR2/3 with PDZ domains regulates hippocampal long-term depression. Proc Natl Acad Sci USA 98:11725-11730.

Liu SJ, Cull-Candy SG (2005) Subunit interaction with PICK and GRIP controls $\mathrm{Ca}^{2+}$ permeability of AMPARs at cerebellar synapses. Nat Neurosci 8:768-775.

Lu W, Ziff EB (2005) PICK1 interacts with ABP/ GRIP to regulate AMPA receptor trafficking. Neuron 47:407-421.

Malinow R, Malenka RC (2002) AMPA receptor trafficking and synaptic plasticity. Annu Rev Neurosci 25:103-126.

Matsuda S, Mikawa S, Hirai H (1999) Phosphorylation of serine- 880 in GluR2 by protein kinase C prevents its $\mathrm{C}$ terminus from binding with glutamate receptor-interacting protein. J Neurochem 73:1765-1768.

Matsuda S, Launey T, Mikawa S, Hirai H (2000) Disruption of AMPA receptor GluR2 clusters following long-term depression induction in cerebellar Purkinje neurons. EMBO J 19:2765-2774.

Perez JL, Khatri L, Chang C, Srivastava S, Osten P, Ziff EB (2001) PICK1 targets activated protein kinase $\mathrm{C} \alpha$ to AMPA receptor clusters in spines of hippocampal neurons and reduces surface levels of the AMPA-type glutamate receptor subunit 2. J Neurosci 21:5417-5428.

Peter BJ, Kent HM, Mills IG, Vallis Y, Butler PJ, Evans PR, McMahon HT (2004) BAR domains as sensors of membrane curvature: the amphiphysin BAR structure. Science 303:495-499.

Pietropaolo M, Castano L, Babu S, Buelow R, Kuo YL, Martin S, Martin A, Powers AC, Prochazka M, Naggert J, et al (1993) Islet cell autoantigen $69 \mathrm{kD}$ (ICA69). Molecular cloning and characterization of a novel diabetes-associated autoantigen. J Clin Invest 92:359-371.

Pilon M, Peng XR, Spence AM, Plasterk RH, Dosch HM (2000) The diabetes autoantigen ICA69 and its Caenorhabditis elegans homologue, ric19 , are conserved regulators of neuroendocrine secretion. Mol Biol Cell 11:3277-3288.

Ren G, Vajjhala P, Lee JS, Winsor B, Munn AL (2006) The BAR domain proteins: molding membranes in fission, fusion, and phagy. Microbiol Mol Biol Rev 70:37-120.

Song I, Huganir RL (2002) Regulation of AMPA receptors during synaptic plasticity. Trends Neuro-

sci 25:578-588.

Spitzenberger F, Pietropaolo S, Verkade P, Habermann B, Lacas-Gervais S, Mziaut H, Pietropaolo M, Solimena M (2003) Islet cell autoantigen of 
$69 \mathrm{kDa}$ is an arfaptin-related protein associated with the Golgi complex of insulinoma INS-1 cells. J Biol Chem 278:26166-26173.

Steinberg JP, Takamiya K, Shen Y, Xia J, Rubio ME, Yu S, Jin W, Thomas GM, Linden DJ, Huganir RL (2006) Targeted in vivo mutations of the AMPA receptor subunit GluR2 and its interacting protein PICK1 eliminate cerebellar long-term depression. Neuron 49:845-860.

Tarricone C, Xiao B, Justin N, Walker PA, Rittinger K, Gamblin SJ, Smerdon SJ (2001) The structural basis of Arfaptin-mediated cross-talk between Rac and Arf signalling pathways. Nature 411:215-219.

Terashima A, Cotton L, Dev KK, Meyer G, Zaman S, Duprat F, Henley JM, Collingridge GL, Isaac JT (2004) Regulation of synaptic strength and AMPA receptor subunit composition by PICK1. J Neurosci 24:5381-5390.

Winer S, Astsaturov I, Gaedigk R, Hammond-McKibben D, Pilon M, Song A,
Kubiak V, Karges W, Arpaia E, McKerlie C, Zucker P, Singh B, Dosch HM (2002a) ICA69(null) nonobese diabetic mice develop diabetes, but resist disease acceleration by cyclophosphamide. J Immunol 168:475-482.

Winer S, Astsaturov I, Cheung R, Tsui H, Song A, Gaedigk R, Winer D, Sampson A, McKerlie C, Bookman A, Dosch HM (2002b)Primary Sjogren's syndrome and deficiency of ICA69. Lancet 360:1063-1069.

Xia J, Zhang X, Staudinger J, Huganir RL (1999) Clustering of AMPA receptors by the synaptic PDZ domain-containing protein PICK1. Neuron 22:179-187.

Xia J, Chung HJ, Wihler C, Huganir RL, Linden DJ (2000) Cerebellar longterm depression requires PKC-regulated interactions between GluR2/3 and PDZ domain-containing proteins. Neuron 28:499-510.

Xu J, Xia J (2007) Structure and function of PICK1. Neurosignals 15:190-201. 